\title{
The Stability analysis for a kind of Impulsive Hopfield
}

\section{cellular neural networks}

\author{
YanLing $\mathrm{Li}^{1, \mathrm{a}}$,Man Hua ${ }^{1, \mathrm{~b}}$ \\ ${ }^{1}$ School of Computer Science, Civil Aviation Flight University of China, GuangHan, \\ Sichuan,618307, China

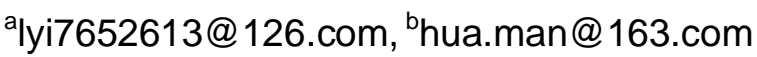

Keywords: CNNs, periodic solution, impulses, hopfield, existence, stability.

Abstract. As an important tool to study practical problems of biology, engineering and image processing, the cellular neural networks (CNNs) has caused more and more attention. In this paper, by means of iterative analysis, the existence of periodic solution and the uniform stability of the equilibrium point of Hopfield cellular neural networks with impulsive effects are considered. Some new results are obtained.

\section{Introduction}

Cellular neural networks (CNNS) is formed by many units called cells, the structure of the CNN is similar to that found in cellular automata, namely, any cell in a cellular neural network is connected only to its neighbor cells. A cell contains linear and non-linear circuit elements, which typically are linear capacitors, linear resistors, linear and nonlinear controlled sources, and independent sources. The circuit diagram and connection pattern modelling a CNN can be found in $[1,2]$. Recently, due to the CNNs' applicability in image and signal processing, vision, pattern recognition and optimization, which have been paid much attention. Extraordinarily, the stability and equilibrium properties of cellular neural networks introduced in [3] have been investigated by many researches [4-6] where most of the results derive the conditions for uniqueness and global asymptotic stability of the equilibrium point for CNNs.

In this paper, we are concerned with the existence of periodic solution and the uniform stability of the equilibrium point for Hopfield CNNs with impulsive effects. Different from most of the existing methods, we employ the iterative analysis method. Several previous results are improved and generalized.

\section{Preliminaries}

In this paper, we consider the following Hopfield CNNs with impulsive effects:

$$
\left\{\begin{array}{lc}
C_{i} \frac{d x_{i}(t)}{d t}=-\frac{x_{i}(t)}{R_{i}}+\sum_{j=1}^{n} a_{i j} f_{j}\left(x_{j}(t)\right)+J_{i}, & t \in J_{1}, \\
\Delta x_{i}\left(t_{k}\right)=x_{i}\left(t_{k}^{+}\right)-x_{i}\left(t_{k}^{-}\right)=H_{i k} x_{i}\left(t_{k}\right), & t=t_{k}, \\
x_{i}(t)=\varphi_{i}(t), & t \in(-\infty, 0],
\end{array}\right.
$$

where $a_{i j} \in R, i, j=1,2, \mathrm{~L}, \boldsymbol{n}, \boldsymbol{J}=[0,+\infty), \quad 0=\boldsymbol{t}_{0}<\boldsymbol{t}_{1}<\boldsymbol{t}_{2}<\mathrm{L}, \boldsymbol{J}^{\prime}=\boldsymbol{J}-\left\{\boldsymbol{t}_{1}, \boldsymbol{t}_{2}, \mathrm{~L}\right\}, \boldsymbol{H}_{i k} \in \boldsymbol{C}(\boldsymbol{R}, \boldsymbol{R})$, 
$\boldsymbol{k} \in \boldsymbol{Z}^{+}, \lim _{\boldsymbol{k} \rightarrow \infty} \boldsymbol{t}_{\boldsymbol{k}}=\infty, \varphi_{i}(\boldsymbol{t})$ is a real-valued continuous function defined on $(-\infty, 0]$. The positive constants $C i$ and $R i$ are the neuron amplifier imput capacitance and resistance, respectively; $J_{i}$ is the constant input from outside of the network, $n$ denotes the number of units in a neural network, $x_{i}(t)$ denotes the state of the $i$ th unit at time $t$ and is a continuous T-periodic function, $f_{\mathrm{j}}\left(x_{\mathrm{j}}(t)\right)$ denotes the output of the $j$ th unit at time $\mathrm{t}, a_{\mathrm{ij}}$ denotes the strength of the $j$ th unit on the $i$ th unit at time $t$, $\Delta x_{i}\left(t_{k}\right)$ corresponds to the abrupt changes of the state at fixed impulsive moment $t_{\mathrm{k}}$.

Let $\boldsymbol{J}_{1}=[0, \boldsymbol{T}], \boldsymbol{J}_{1} \cap \boldsymbol{t}_{\boldsymbol{k}}=\left\{\boldsymbol{t}_{1}, \boldsymbol{t}_{2}, \mathrm{~L}, \boldsymbol{t}_{p}\right\}, \boldsymbol{P C}\left(\boldsymbol{J}_{1}, \boldsymbol{R}\right)=\left\{\boldsymbol{x}_{\boldsymbol{i}}: \boldsymbol{J}_{1} \rightarrow \boldsymbol{R} ; x_{\mathrm{i}}(\mathrm{t})\right.$ is continuous everywhere except for some $t_{\mathrm{k}}$ at which $\boldsymbol{x}_{\boldsymbol{i}}\left(\boldsymbol{t}_{k}^{+}\right)$and $\boldsymbol{x}_{\boldsymbol{i}}\left(\boldsymbol{t}_{\boldsymbol{k}}^{-}\right)$exist and $\boldsymbol{x}_{\boldsymbol{i}}\left(\boldsymbol{t}_{\boldsymbol{k}}\right)=\boldsymbol{x}_{\boldsymbol{i}}\left(\boldsymbol{t}_{\boldsymbol{k}}^{-}\right), \boldsymbol{k}=1,2, \mathrm{~L}, \boldsymbol{p}$.. With norm $\left\|\boldsymbol{x}_{i}\right\|_{P}=\sup \left\{\left|\boldsymbol{x}_{\boldsymbol{i}}(\boldsymbol{t})\right|: \boldsymbol{t} \in \boldsymbol{J}_{1}\right\}$, then $\mathrm{P}$ is a Banach space.

We call constant vector $x^{*}=\left(x_{1}^{*}, x_{2}^{*}, \mathrm{~L}, x_{n}^{*}\right)^{T}$ as the equilibrium point of system (2.1), if it satisfies the following equation:

$$
\left\{\begin{array}{l}
\frac{1}{\boldsymbol{R}_{i}} \boldsymbol{x}_{i}^{*}=\sum_{j=1}^{n} a_{i j} f_{j}\left(x_{i}^{*}\right)+J_{i}, \quad t \neq t_{k}, \\
\boldsymbol{H}_{i k}\left(x_{i}^{*}\right)=0, \quad t=t_{k} .
\end{array}\right.
$$

In this paper, we assume that some conditions are satisfied so that the equilibrium point of system (2.1) does exist.

In order to prove the stability of the equilibrium point of system (2.1), we just need to prove the stability of zero solution of following system:

$$
\begin{cases}C_{i} y_{i}^{\prime}(t)=-\frac{y_{i}(t)}{R_{i}}+\sum_{j=1}^{n} a_{i j} g_{j}\left(y_{j}(t)\right), & t \in J^{\prime}, \\ \Delta y_{i}\left(t_{k}\right)=G_{i k}\left(y_{i}\left(t_{k}\right)\right), & t=t_{k}, \\ y_{i}(t)=\phi_{i}(t), & t \in(-\infty, 0] .\end{cases}
$$

where $g_{j}\left(y_{j}(t)\right)=f_{j}\left(y_{j}(t)+x_{j}^{*}\right)-f_{j}\left(x_{j}^{*}\right), G_{i k}\left(y_{i}\left(t_{k}\right)\right)=H_{i k}\left(y_{i}\left(t_{k}\right)+x_{i}^{*}\right), \phi_{i}(t)=\varphi_{i}(t)-x_{i}^{*}$. Let

$$
\|\Phi\|=\max \left\{\sup _{-\infty<t \leq 0}\left|\phi_{i}(t)\right|, \boldsymbol{i}=1,2, \mathrm{~L}, \boldsymbol{n}\right\}, \quad\|\boldsymbol{y}\|=\max \left\{\sup _{0 \leq t<+\infty}\left|\boldsymbol{y}_{\boldsymbol{i}}(\boldsymbol{t})\right|, \boldsymbol{i}=1,2, \mathrm{~L}, \boldsymbol{n}\right\} .
$$

Definition A piecewise continuous function $\boldsymbol{x}(\boldsymbol{t}) \in \boldsymbol{P}_{0}:[0, \boldsymbol{T}] \rightarrow \boldsymbol{R}^{n}$ is called a T-periodic solution of Eq.(2.1), if

(1) $x(\mathrm{t})$ satisfies Eq.(2.1) for $t \in(-\infty, T]$;

(2) $x(t)=x(t+T)$ for $t \in \boldsymbol{R}^{+}$; 
(3) $x(\mathrm{t})$ is continuous at $t \neq \boldsymbol{t}_{k}, \boldsymbol{x}\left(\boldsymbol{t}_{\boldsymbol{k}}\right)=\boldsymbol{x}\left(\boldsymbol{t}_{k}^{-}\right)$, and $\boldsymbol{x}\left(\boldsymbol{t}_{k}^{+}\right)$exist for $\forall t_{k} \in J_{1} \cap\left\{\boldsymbol{t}_{k}\right\}$.

The following are the basic hypotheses:

$\left(H_{1}\right)$ There exist constants $L_{\mathrm{i}}>0$ such that $\left|f_{i}\left(\boldsymbol{x}_{1}\right)-\boldsymbol{f}_{i}\left(\boldsymbol{x}_{2}\right)\right| \leq \boldsymbol{L}_{i}\left|\boldsymbol{x}_{1}-\boldsymbol{x}_{2}\right|$;

$\left(H_{2}\right)$ There exist constants $\mathrm{q}_{\mathrm{ik}}>0$ such that $\left|\boldsymbol{H}_{i k}\left(\boldsymbol{x}_{1}\right)-\boldsymbol{H}_{\boldsymbol{i k}}\left(\boldsymbol{x}_{2}\right)\right| \leq \boldsymbol{q}_{i k}\left|\boldsymbol{x}_{1}-\boldsymbol{x}_{2}\right|$;

We denote:

$$
\begin{gathered}
a=\max _{i, j=1, \mathrm{~L}, n}\left\{\left|a_{i j}\right|\right\}, \quad D_{i}=T L_{i}, \quad D^{+}=\max _{i, j=1, \mathrm{~L}, n}\left\{D_{i}\right\}, \quad Q_{i}=\sum_{k=1}^{p} q_{i k} \\
F=\min _{i=1, \mathrm{~L}, n}\left\{\left(1-e^{-\frac{1}{C_{i} R_{i}} T}-Q_{i} \mid C_{i}\right\}, A=\max _{i j=1, \mathrm{~L}, n}\left\{\frac{D^{+}+Q_{i} C_{i}}{\left(1-e^{-\frac{1}{C_{i} R_{i}}}\right) C_{i}}\right\}, D=\max _{i=1, \mathrm{~L}, n}\left\{\frac{\sum_{j=1}^{n}\left|a_{i j}\right| D^{+}}{\left(1-e^{-\frac{1}{C_{i} R_{i}}}\right) C_{i}}\right\} .\right. \\
\left(H_{3}\right) \quad 0<A<1, \quad 0<\frac{n a D^{+}}{F}<1 .
\end{gathered}
$$

Lemma 2.1 Let $y(t)=\left[y_{1}(t), y_{2}(t), \mathrm{L}, y_{n}(t)\right]^{T}$ be a T-periodic solution of Eq.(2.2), then it can be presented as

$$
y_{i}(t)=\int_{0}^{T} \frac{g_{i}(t, s)}{C_{i}} \sum_{j=1}^{n} a_{i j} g_{j}\left(y_{j}(s)\right) d s+\sum_{k=1}^{p} g_{i}\left(t, t_{k}\right) G_{i k}\left(y_{i}\left(t_{k}\right)\right), \quad t \in J_{1},
$$

where

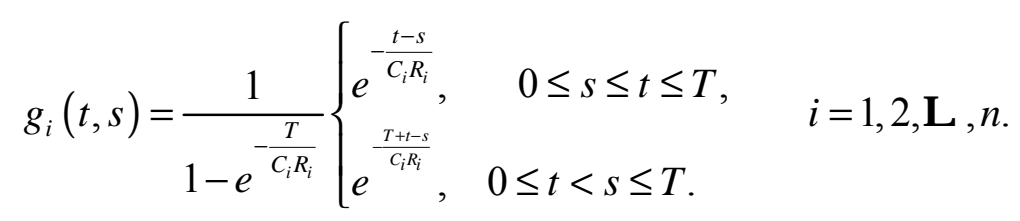

\section{Main results}

Theorem 3.1 Suppose that hypotheses $\left(\mathrm{H}_{1}\right)-\left(\mathrm{H}_{3}\right)$ hold, then the problem (2.2) has a unique $T$-periodic solution $y(t)=\left[\boldsymbol{y}_{1}(\boldsymbol{t}), \boldsymbol{y}_{2}(\boldsymbol{t}), \mathrm{L}, \boldsymbol{y}_{\boldsymbol{n}}(\boldsymbol{t})\right]^{T}$ on[0,T], and

$$
\left\|y_{i}\right\|_{P} \leq \frac{2 D}{1-A}\|\Phi\|, i=1,2, \mathrm{~L}, n
$$

Proof. We define the iteration

$$
y_{i}^{(m)}(t)=\left\{\begin{array}{l}
\int_{0}^{T} \frac{g_{i}(t, s)}{C_{i}} \sum_{j=1}^{n} a_{i j} g_{j}\left(y_{j}^{(m-1)}(s)\right) d s+\sum_{k=1}^{p} g_{i}\left(t, t_{k}\right) G_{i k}\left(y_{i}^{(m-1)}\left(t_{k}\right)\right), \quad t \in J_{1}, \\
\|\Phi\|, \quad t \in(-\infty, 0]
\end{array}\right.
$$




$$
\begin{aligned}
& y_{i}^{(0)}(t)=\left\{\begin{array}{l}
\int_{0}^{T} \frac{g_{i}(t, s)}{C_{i}} \sum_{j=1}^{n} a_{i j} g_{j}\left(y_{j}(0)\right) d s, \\
\|\Phi\|, \\
\| \in(-\infty, 0] .
\end{array}\right. \\
& \left|y_{i}^{(1)}(t)-y_{i}^{(0)}(t)\right| \leq \int_{0}^{T} \frac{\left|g_{i}(t, s)\right|}{C_{i}} \sum_{j=1}^{n}\left|a_{i j}\right| L_{j}\left|y_{j}^{(0)}(s)-y_{j}(0)\right| d s+\sum_{k=1}^{p} q_{i k}\left|g_{i}\left(t, t_{k}\right)\right||| y_{i}^{(0)}\left(t_{k}\right) \mid \|, \\
& \left\|y_{i}^{(1)}-y_{i}^{(0)}\right\|_{P} \leq \frac{D\|\Phi\|+\|\Phi\|}{\left(1-e^{-\frac{T}{C_{i} R_{i}}}\right) C_{i}} \sum_{j=1}^{n}\left|a_{i j}\right| D^{+}+\frac{Q_{i} D\|\Phi\|}{1-e^{-\frac{T}{C_{i} R_{i}}}} \leq(A+1) D\|\Phi\| . \\
& \left\|y_{i}^{(2)}-y_{i}^{(1)}\right\|_{P} \leq A(A+1) D\|\Phi\| . \quad\left\|y_{i}^{(3)}-y_{i}^{(2)}\right\|_{P} \leq A^{2}(A+1) D\|\Phi\| .
\end{aligned}
$$

Again, using induction, we can get that

$$
\begin{gathered}
\left\|y_{i}^{(m+1)}-y_{i}^{(m)}\right\|_{P} \leq A^{m}(A+1) D\|\Phi\|, \quad m=0,1, \mathrm{~L}, \quad i=1,2, \mathrm{~L}, n . \\
\left\|y_{i}^{(n+1)}\right\|_{P} \leq \sum_{m=0}^{n}\left|y_{i}^{(m+1)}(t)-y_{i}^{(m)}(t)\right|+\left|y_{i}^{(0)}(t)\right| \leq \frac{2 D\|\Phi\|}{1-A} .
\end{gathered}
$$

For any $\boldsymbol{p} \in \boldsymbol{N}, \boldsymbol{n}+\boldsymbol{p} \geq \boldsymbol{n}, \boldsymbol{i}=1,2, \mathrm{~L}, \boldsymbol{n}$, we have $\left|y_{i}^{(n+p)}(t)-y_{i}^{(n)}(t)\right| \leq \frac{A^{n}}{1-A}(1+A) D\|\Phi\|$.

Therefore, the sequence $\left\{y_{i}^{(m)}(t)\right\}$ is uniformly convergent on [0,T], let $\lim _{m \rightarrow \infty} y_{i}^{(m)}(t)=y_{i}(t)$, obviously, $y_{i}(t)$ is a T-periodic solution to the initial value problem (2.2), which satisfies the inequality (3.1). This completes the proof of Theorem 3.1.

Theorem 3.2 Suppose that the hypotheses $\left(\mathrm{H}_{1}\right)-\left(\mathrm{H}_{3}\right)$ hold, then the zero solution of the initial value problem (2.2) is uniformly stable.

Theorem 3.3 Suppose that the hypotheses $\left(\mathrm{H}_{1}\right)-\left(\mathrm{H}_{3}\right)$ hold, then the equilibrium point of the initial value problem (2.1) is uniformly stable.

\section{References}

[1] L. O. Chua and L. Yang,Cellular neural networks: theory and applications. IEEE Trans.Circuits and Syst., 35, pp. 1257-1290, 1988.

[2] Yongqing Yang and Jinde Cao,Stability and periodicity in delayed cellular neural networks with impulsive effects. Nonlinear Analysis: Real Word Applications, 8, pp. 362-374, 2007.

[3] P. L. Venetianer and T. Roska,Image compression by cellular neural networks. IEEE Trans. Circuits Syst., 45, pp. 205-215, 1998.

[4] H. Akca, R. Alassar, V. Covachev, Z. Covachev and E. A. Zahrani,Continuous-time additive Hopfield-type neural networks with impulses. J. Math. Anal. Appl., 290, pp. 436-451, 2004.

[5] Z. Guan, J. Lam and G. Chen,On impulsive autoassociative neural networks. Neural Networks, 13, pp. 63-69, 2000.

[6] Z. Guan and G. Chen,On delayed impulsive Hopfield neural networks. Neural Networks, 12, pp. 273-280, 1999. 